\title{
La necesaria perspectiva de género para el análisis de problemas de salud
}

\author{
The necessary gender perspective for the analysis of health problems \\ Jorge A. Álvarez-Díaz* \\ Departamento de Atención a la Salud, Universidad Autónoma Metropolitana, Unidad Xochimilco, Cuidad de México, México
}

\section{Resumen}

Este trabajo inicia con la diferencia entre sexo y género, para mostrar que los contrastes en el campo de la salud entre hombres y mujeres pueden explicarse por la construcción de género de consultantes y de proveedores de servicios de salud, por la estructura de los propios servicios de salud (que refleja también construcciones de género) y por la reproducción de patrones de género a través de la educación y la investigación. El trabajo muestra la necesidad de incluir la perspectiva de género en la investigación en salud.

Palabras Clave: Perspectiva de género. Ciencias sociales. Interseccionalidad.

\begin{abstract}
This work begins with the difference between sex and gender, then show that the contrasts in the field of health between men and women can be explained: by the construction of gender of consultants and health service providers, by the structure of the services themselves of health (which also reflects gender constructions), and by the reproduction of gender patterns through education and research. The work shows the need to include a gender perspective in health research.
\end{abstract}

Key Words: Perspective of gender. Social sciences. Intersectionality.

\section{Introducción}

La primera distinción que debe establecerse es entre «sexo» y "género». El sexo corresponde al conjunto de características fenotípicas que expresan el genotipo heredado y que colocan al ser humano en algún punto de un continuo, el cual tiene en los extremos individuos con potencial reproductivo complementario (esto es, el macho y la hembra de la especie humana). Tras la unión de espermatozoide y ovocito, y la generación del cigoto, se complementa el juego de gonosomas sexuales, constituidos por genes, de tal modo que el efecto fenotípico dependerá de la presencia o ausencia de algunos genes clave para la diferenciación. Dependiendo de la genómica recibida se expresarán las transcriptómicas, generando proteómicas y sus metabolomas, de modo tal que el desarrollo embrionario irá expresando el fenotipo a

\footnotetext{
Correspondencia:

*Jorge A. Álvarez-Díaz

Calzada del Hueso, 1100

Col. Villa Quietud, Del. Coyoacán

Fecha de recepción: 02-11-2018

C.P. 04960, Ciudad de México, México

E-mail: bioetica_reproductiva@ hotmail.com

0009-7411/@ 2019 Academia Mexicana de Cirugía. Publicado por Permanyer. Este es un artículo open access bajo la licencia CC BY-NC-ND (http://creativecommons.org/licenses/by-nc-nd/4.0/).

Cir Cir. 2020;88(3):383-388

Contents available at PubMed www.cirugiaycirujanos.com
} 
través de la gónada primitiva y el resto de estructuras de los órganos sexuales pélvicos internos y externos, la síntesis de hormonas consecuente a la diferenciación y maduración de la gónada, y con ello el impacto en órganos diana, particularmente el encéfalo y el aparato musculoesquelético. Esta sucesión de eventos del desarrollo embrionario da lugar al sexo genotípico (cromosómico, genético) y al sexo fenotípico (gonadal, hormonal, encefálico, morfológico). Sin embargo, estas posibilidades humanas no siempre han quedado claras. Lo cultural ha sido identificar a «hombres» $y$ «mujeres» desde el fenotipo, suponiendo que solo existen dos sexos desde la biología; si algún fenotipo no estaba del todo claro para el observador, resultaba una anomalía, un «trastorno de la diferenciación sexual», que colocaba al fenotipo de un ser humano en un «estado intersexual». Bajo la lógica de dos sexos, el resto de las posibilidades suponían un problema y se localizaban "entre dos sexos", y de ahí esta nomenclatura. Entender que los procesos de diferenciación sexual son diversos introduce la idea de que hay diversos sexos y no solo dos.

Por otra parte, el género tiene algunos problemas para teorizarse. Primero, porque en lengua inglesa el término gender no significa exactamente lo mismo que se expresa con la palabra española "género». En inglés, gender hace referencia a características tanto biológicas como sociales. Por ejemplo, en el diccionario Oxford se define el género de la siguiente manera: "Cualquiera de los dos sexos (masculino y femenino), especialmente cuando se considera con referencia a las diferencias sociales y culturales en lugar de las biológicas. El término también se usa de manera más amplia para denotar un rango de identidades que no se corresponden con las ideas establecidas de hombres y mujeres»'. El desarrollo del concepto en lengua española no ha sido en ese sentido, y «género» se refiere a la construcción sociocultural que supone una serie de roles y actitudes que se espera deberían presentar hombres y mujeres, y que por ello son impuestas y reforzadas a través de diferentes estructuras sociales (familia, escuela, trabajo, etc.). Como la visión tradicional ha sido la existencia de dos sexos, se han supuesto dos construcciones culturales posibles: la masculina (lo esperado y deseable en roles y actitudes de hombres) y la femenina (lo esperado y deseado en roles y actitudes de mujeres). La división entre lo masculino y lo femenino ha sido fundamentalmente el ejercicio del poder: mientras a lo masculino le corresponde la dominación (siguiendo a Pierre Bordieu), a lo femenino le corresponde el cautiverio (siguiendo a Marcela Lagarde). Esta visión es la más simple respecto al género y presenta una serie de problemas. Primero, si no existen solo dos sexos, como ya se aclaró, no pueden establecerse solo dos construcciones. Segundo, entender el género de forma dicotómica con la distribución asimétrica del poder (a través del «cisheteropatriarcado» o simplemente «patriarcado») Ileva a que algunos sujetos sigan lo esperado, deseado o impuesto socioculturalmente, pero otros no. Así, habría entonces «masculinidades», una «masculinidad hegemónica» (siguiendo a Antonio Gramsci) y «masculinidades disidentes». Así mismo, habría una «feminidad hegemónica» y «feminidades disidentes». En realidad, hay muchas formas sociales de ser hombre, como muchas formas de ser mujer; valdría decir, simplemente, «masculinidades» y «feminidades». Existen otras formas de explicar el posicionamiento respecto del género binario, pudiendo ser bigenéricas o de género fluido, o bien formas no binarias como el trigenerismo o pangenerismo, e incluso el posicionamiento queer (donde no cabe ninguna categoría rígida).

En adelante hablaremos de forma dicotómica respecto de «hombres y mujeres» y «masculino y femenino» por hacer más sencilla la exposición. Iniciar con la distinción entre sexo y género es relevante por varios aspectos. En la práctica clínica, tradicionalmente se ha entendido que la biología tiene una correlación o una relación causal con la presencia o la ausencia de enfermedades. Por ello, desde el punto de vista de la epidemiología se ha buscado un registro con variables como el sexo. La salud colectiva ha criticado el reduccionismo del modelo biomédico, resaltando que el análisis histórico y sociocultural de las prácticas e instituciones de salud muestran que hay dimensiones simbólicas, políticas, socioeconómicas, bioéticas, etc., en los procesos de salud/enfermedad. Así, reconocer que hay determinantes históricos y socioculturales, además de los biomédicos, para los procesos de salud/enfermedad, muestra qué tan necesario es tratar tanto el sexo como el género.

\section{Género, medicina y salud}

Existe literatura especializada en lengua inglesa que indica que "las diferencias de género en salud resultan de una interacción compleja de diferencias básicas en biología»². Sin embargo, no será ese el sentido al hablar en adelante del género, sino 
entendiéndolo como una construcción histórica y sociocultural. Así, las diferencias en el campo de la salud observadas entre hombres y mujeres pueden explicarse desde diferentes vertientes: por la construcción de género de consultantes, por la construcción de género de proveedores de servicios de salud y por la estructura de los propios servicios de salud, que refleja también construcciones respecto al género. Parte de la explicación también radica en la reproducción de patrones de género en la educación y la investigación.

Las diferencias en relación con el género de las personas consultantes están algo más documentadas. Las mujeres interactúan con los servicios de salud con más frecuencia, tanto para la prevención como para el tratamiento ${ }^{3}$. La esperanza de vida de las mujeres, por lo general, es mayor que la de los hombres; además, tienen más probabilidades de sobrevivir que sus cónyuges ${ }^{4}$, son más propensas que los hombres a tener discapacidades funcionales en edades avanzadas ${ }^{5} \mathrm{y}$, por ello, son las usuarias principales de seguimiento y cuidados a largo plazo, incluidos los hogares de personas ancianas ${ }^{6}$. En la mayoría de los indicadores, las mujeres utilizan más servicios de salud7. Una posible explicación de ese mayor uso de los servicios es el llamado "comportamiento de búsqueda de salud", un patrón conductual aprendido en las mujeres por el rol de cuidado. Según esta explicación, las mujeres usan más servicios porque han sido socializadas para reconocer y articular signos y síntomas corporales para percibir una mayor necesidad de atención y buscar la ayuda de otros ${ }^{8}$. La socialización de las mujeres para el comportamiento de búsqueda de salud puede reflejar la expectativa cultural de que las mujeres asuman responsabilidades de cuidado en la familia. Si a las mujeres les ha correspondido el rol social tradicional de cuidado, se esperaría que buscaran información de salud más activamente y que además fueran más tenaces para superar las barreras de acceso. De acuerdo con revisiones realizadas desde mediados de la década de 1980, los riesgos diferenciales adquiridos por el género tienen esa relación estrecha con los estilos de vida y las prácticas preventivas de salud $^{9}$.

También existen diferencias en relación con el género de proveedores de servicios de salud, algo menos estudiadas. Diversos estudios han demostrado que los hombres y las mujeres difieren en los estilos de comunicación; las posibles diferencias entre médicos y médicas han mostrado distintos tipos de comunicación médico-paciente, de proceso de diagnóstico y de tratamiento. El estilo de comunicación de las médicas está más orientado al paciente que el de los médicos, y médicos y médicas difieren en el uso de pruebas adicionales; en particular, los exámenes íntimos (tactos vaginales o tactos rectales) se realizan con menos frecuencia en pacientes del sexo opuesto. Los médicos recetan medicamentos con más frecuencia; en especial, los sedantes son prescritos con más frecuencia por médicos a mujeres. Además, estudiantes de medicina hombres y mujeres socializan de manera diferente, y la socialización profesional no supera las diferencias en los roles de género. Con todo ello puede apreciarse que el rol profesional de quien ejerce la medicina no es neutral en términos de género ${ }^{10}$. No se trata de un mero asunto subjetivo o de confort. Se empieza a estudiar si el género tiene repercusiones en la calidad objetiva de la atención, y parece ser que sí. En un estudio muy reciente se analizó el efecto del sexo del cirujano en los resultados posoperatorios de pacientes sometidos a procedimientos quirúrgicos comunes. Mediante una cohorte retrospectiva, pacientes sometidos a uno de 25 procedimientos quirúrgicos realizados por una cirujana se parearon con pacientes sometidos a la misma operación por un cirujano (por edad, sexo y comorbilidad del paciente, por volumen de trabajo y edad del cirujano, y por hospital). Se evaluaron la mortalidad, las readmisiones y las complicaciones. Los pacientes tratados por cirujanas tuvieron una menor mortalidad a los 30 días (estadísticamente significativa). Los pacientes tratados por cirujanas tuvieron resultados quirúrgicos similares (duración de la estadía, complicaciones y readmisión) a los de aquellos tratados por cirujanos. Estos hallazgos respaldan la necesidad de la inclusión de la perspectiva de género en la formación de los prestadores de servicios de salud ${ }^{11}$.

También se encuentran diferencias de género atribuidas a los servicios de salud. Este tipo de servicios, como toda estructura histórica y sociocultural, también responde a la sociedad a la que sirven. Si en los roles sociales tradicionales los hombres se desempeñan como proveedores y las mujeres como cuidadoras, a los hombres les corresponde el rol de trabajadores. En muchos lugares sigue estando asociado el estatus de trabajador a poder tener acceso a servicios de salud ${ }^{12}$. Esto tiene otras consecuencias, como por ejemplo que las mujeres empleen en mayor proporción los recursos económicos propios como fuente de financiación para sus problemas de 
salud. Una investigación en los principales 50 centros médicos académicos de los EE.UU. encontró que un $32 \%$ ofrecía servicios específicos de cuidado de la salud orientados para hombres, en tanto que un $98 \%$ ofrecía servicios de salud para mujeres ${ }^{13}$. Para poder brindar servicios de salud de forma equitativa debe haber una mayor comprensión de las necesidades por género y no solo por la consideración del sexo ${ }^{14}$.

El hecho de que existan estas diferencias por género en consultantes, en proveedores de servicios de salud y en la estructura de los servicios puede explicarse en parte por aspectos educativos. Lo común en planes y programas de estudio es que la perspectiva de género esté ausente ${ }^{15}$. En epidemiología suele existir un control sistemático de algunas variables, como la edad y el sexo, pero es infrecuente que se realicen consideraciones de género ${ }^{16}$. Muchas veces, tal vez por la influencia del término gender, al hacer un análisis atendiendo al sexo se cree que se están estudiando aspectos relacionados con el género.

Max Weber consideró que la ciencia es y debe ser neutral. Actualmente, ese punto de vista es insostenible: todo científico valora, ya sea científico social o científico natural. Las valoraciones que tienen que ver con el género también están presentes. Se ha estudiado que al explicar procesos biológicos como la fecundación (que aparentemente tendría que ver solo con el sexo y la pura biología, y no con el género ni con cuestiones socioculturales) se hacen consideraciones en las que se reproducen estereotipos de género ${ }^{17}$. Considerar al ovocito como una «bella durmiente» (el rol genérico de cautiverio), esperando a que un espermatozoide entre muchos millones, «el más fuerte», llegue y la conquiste después de «vencer» al resto (con el rol genérico de dominio), muestra como mínimo que hay metáforas que tienen sesgos de género ${ }^{18}$. Sistematizando este tipo de conocimiento, parece claro que a lo largo de la historia, e incluso en el mundo contemporáneo, esas consideraciones culturales se han filtrado en el propio proceso de construcción de la ciencia, con sesgos antropocéntricos, etnocéntricos y androcéntricos ${ }^{19}$.

Esto también debe ser considerado por la medicina y otras disciplinas relacionadas con la salud. Aun en lengua inglesa, entendiendo por gender categorías biológicas, es posible darse cuenta de que la medicina basada en evidencias aporta poca evidencia sobre diferencias por sexo ${ }^{20,21}$. Se ha visto que no se realizan igual la valoración de resultados y el análisis de datos en salud si el investigador principal es un hombre o una mujer ${ }^{22}$.

\section{¿Hacia dónde seguir?}

Si bien siguen surgiendo trabajos que intentan hacer aproximaciones a procesos de salud/enfermedad con perspectiva de género y no meramente estableciendo diferencias por sexo, también es cierto que existen otras consideraciones que hacer sobre las estructuras sociales, como por ejemplo la clase social. Este concepto ha sido casi ignorado al tratar los temas de medicina y salud ${ }^{23}$. Probablemente, uno de los conceptos más ricos para atender este punto sea una aportación feminista: la «interseccionalidad». Este concepto intenta aportar marcos para estudiar mecanismos de discriminación a través de interacciones de subordinación, y no solo la relación entre dominio y cautiverio de lo masculino sobre lo femenino en el género, sino también atendiendo a aspectos como clase social, identidad sexo-genérica, orientación sexual, etnicidad, religión, origen nacional, discapacidad, etc. ${ }^{24,25}$. Todo esto muestra que la investigación desde la perspectiva de género en salud debe seguir desafiando el etnocentrismo andronormativo, evidenciando diferencias por cuestiones de género y desvelando las consecuencias para los procesos de salud/enfermedad de las desigualdades de poder establecidas por el género y otras formas de ejercicio del poder en las que exista subordinación ${ }^{26}$.

Para cerrar, un ejemplo estudiado (aunque no lo suficiente) es diabetes mellitus tipo 2 (DM2). México ocupa el segundo lugar en Latinoamérica y sexto en el mundo en cuanto a prevalencia de DM2, con unos 11.5 millones de casos. La revisión más reciente de epidemiología genética evaluó 68 polimorfismos de 41 genes, y 26 de ellos se asociaron con riesgo de DM2 (ABCA1, ADRB3, CAPN10, CDC123/CAMK1D, CDKAL1, CDKN2A/2B, CRP, ELMO1, FTO, HHEX, IGF2BP2, IRS1, JCFQ1, KCNQ1, LOC387761, SIRT1, SLC30A8, TCF7L2 y los genes TNF- $\alpha$ ); en general, 21 de los 41 genes analizados se asociaron con DM2 en población mexicana ${ }^{27}$. Sin embargo, en estudios genéticos se observa un efecto aditivo cuando se consideran variables tales como la edad, la educación, el índice de masa corporal, la ancestría y el sexo ${ }^{28}$. En otro trabajo se buscó evidencia en las investigaciones que presentan desigualdades entre hombres y mujeres que padecen DM2 en cuanto a prevalencia, incidencia, complicaciones y mortalidad, y apenas se encontraron 22 trabajos, de los que seis demostraban diferencia significativa en prevalencia, ocho en control y complicaciones, y ocho en mortalidad. En la mayoría 
de los trabajos no se encontraron efectos atribuibles a factores biológicos, por lo que recomiendan que, para comprender las diferencias, deberían incluirse en las investigaciones indicadores con perspectiva de género ${ }^{29}$.

Si se pasa de la epidemiología genética a la epidemiología social ${ }^{30}$ puede conseguirse un enfoque tanto o más necesario en el caso de la DM2. Esto es porque, en el caso de las enfermedades crónicas, su aparición, desarrollo y desenlace están íntimamente ligados a aspectos psicosociales ${ }^{31}$. Para 2013, la mayor causa de pérdida de años de vida ajustados por discapacidad en México fue la DM2 ${ }^{32}$. Atendiendo a la última Encuesta Nacional de Salud y Nutrición, de 2012, las prevalencias de sobrepeso y obesidad combinadas fue del $71.2 \%$ (lo que supone un aumento del $15.2 \%$ en los últimos 12 años), siendo del $73 \%$ en mujeres y del $69.4 \%$ en los hombres; en cuanto a la obesidad sola, la prevalencia fue del $37.5 \%$ en la mujeres y del $26.8 \%$ en los hombres ${ }^{33}$. Esto tiene un correlato con cambios en los patrones de alimentación, cuyo análisis muestra que entre 1961 y 2009 se redujo la energía derivada de cereales y leguminosas, y aumentó a la par la energía procedente de azúcares, grasas vegetales y alimentos de origen animal. Se considera que la determinación socioeconómica que modificó la dieta y aumentó la mortalidad por diabetes tuvo relación con la apertura comercial del país, la falta de crecimiento económico, el aumento de la desigualdad, el aumento del trabajo informal, el poco apoyo al sector agrícola, la caída de los salarios en relación con la canasta básica (alimentaria y no alimentaria), el aumento del precio relativo de los alimentos saludables respecto a los procesados, y la desregulación del mercado alimentario ${ }^{34}$.

La interseccionalidad mencionada puede mostrarse con la investigación en salud. Ser mujer es un determinante social para padecer DM2 $2^{35}$. Además, ser pobre también es un determinante social para padecer $\mathrm{DM} 2^{36}$. Ser mujer y pobre determina un peor pronóstico en el curso de la DM2 ${ }^{37,38}$. El metaanálisis más reciente (2017) y más amplio (1.7 millones de hombres y mujeres) deja datos muy claros ${ }^{39}$ : el bajo nivel socioeconómico es uno de los indicadores más fuertes de la morbilidad y la mortalidad prematura en todo el mundo. La pobreza acorta la vida más que la obesidad, el alcohol y la hipertensión. La evidencia dice que la desigualdad mata. ¿Interesa del mismo modo la salud del país, tanto la de los pobres como la de los ricos? ¿Interesa del mismo modo la salud de las mujeres que la de los hombres?

\section{Agradecimientos}

Una parte de este trabajo fue presentada en la LII Reunión Ordinaria de la Asociación Mexicana de Escuelas de Salud Pública (AMESP), La reducción del impacto en salud ante emergencias y desastres. Prioridad en la formación de recursos humanos en salud pública, en la conferencia titulada Desastres: bioética, género y voluntariado.

\section{Conflicto de intereses}

El autor declara que durante la elaboración de este trabajo no hubo conflicto de intereses.

\section{Responsabilidades éticas}

Protección de personas y animales. Los autores declaran que para esta investigación no se han realizado experimentos en seres humanos ni en animales.

Confidencialidad de los datos. Los autores declaran que en este artículo no aparecen datos de pacientes.

Derecho a la privacidad y consentimiento informado. Los autores declaran que en este artículo no aparecen datos de pacientes.

\section{Bibliografía}

1. Diccionario Oxford. Entrada: Gender. [Citado el 1 de noviembre de 2018]. Disponible en: https://en.oxforddictionaries.com/definition/gender

2. Gabriel SE. The epidemiology of gender-discrepant illness. Lupus. 1999; 8:339-45.

3. National Center for Health Statistics (NCHS). Health United States, 1995. Hyattsville, MD: Public Health Service; 1996.

4. Horton JA. The women's health data book: a profile of women's health in the United States. Washington DC: Jacobs Institute of Women's Health; 1995.

5. Hibbard JH, Pope CR. Another look at sex differences in the use of medical care: illness orientation and the types of morbidities for which services are used. Women Health. 1986;11:21-36.

6. Garrett JM, Harris RP, Norburn JK, Patrick DL, Danis M. Life-sustaining treatments during terminal illness: who wants what? J Gen Intern Med. 1993;8:361-8.

7. Mechanic D. Sex, illness, illness behavior, and the use of health services. Soc Sci Med. 1978;12:207-14.

8. Hing E, Kovar MG, Rice DP. Sex differences in health and use of medical care: United States, 1979. Vital Health Stat 3. 1983:24:1-55.

9. Verbrugge LM. Gender and health: an update on hypotheses and evidence. J Health Soc Behav. 1985;26:156-82.

10. Lagro-Janssen AL. De geneeskunde is niet genderneutraal: invloed van de sekse van de dokter op de medische zorg. Ned Tijdschr Geneeskd. 2008;152:1141-5.

11. Wallis CJ, Ravi B, Coburn N, Nam RK, Detsky AS, Satkunasivam R. Comparison of postoperative outcomes among patients treated by male and female surgeons: a population based matched cohort study. BMJ. 2017;359:j4366.

12. Guarnizo-Herreño CC, Agudelo $C$. Equidad de género en el acceso a los servicios de salud en Colombia. Rev Salud Publica (Bogota). 2008;10(Suppl 1):44-57.

13. Choy J, Kashanian JA, Sharma V, Masson P, Dupree J, Le B, et al. The men's health center: disparities in gender specific health services among the top 50 "best hospitals" in America. Asian J Urol. 2015;2:170-4.

14. Gómez Gómez E. Género, equidad y acceso a los servicios de salud: una aproximación empírica. Rev Panam Salud Publica. 2002;11:327-34.

15. Wong YL. Gender competencies in the medical curriculum: addressing gender bias in medicine. Asia Pac J Public Health. 2009;21:359-76. 
16. Bolte G. Gender in der epidemiologie. Diskussionsstand und perspektiven. Bundesgesundheitsbl - Gesundheitsforsch - Gesundheitsschutz. 2008;51:3-12.

17. Martin E. The egg and the sperm: how science has constructed a romance based on stereotypical male-female roles. Signs. 1991;16:485-501.

18. Pérez Sedeño E. El sexo de las metáforas. ARBOR Ciencia, Pensamiento y Cultura. 2011;187:99-108.

19. García Dauder S, Pérez Sedeño E. Las "mentiras" científicas sobre las mujeres. Madrid: Catarata; 2017.

20. Holdcroft A. Gender bias in research: how does it affect evidence based medicine? J R Soc Med. 2007;100:2-3.

21. Deidda M, Cadeddu Dessalvi C, Mercuro G. The need of a gender-corrected evidence based medicine. Int J Cardiol. 2018;255:156-7.

22. Polit DF, Beck CT. Gender bias undermines evidence on gender and health. Qual Health Res. 2012;22:1298.

23. Krieger N, Fee E. Man-made medicine and women's health: the biopolitics of sex/gender and race/ethnicity. Int J Health Serv. 1994;24:265-83.

24. La Barbera MC. Interseccionalidad, un "concepto viajero": orígenes, desarrollo e implementación en la Unión Europea. Interdisciplina. 2016; 4:105-22.

25. Viveros Vigoya M. La interseccionalidad: una aproximación situada a la dominación. Debate Feminista. 2016:52:1-17.

26. Hølge-Hazelton B, Malterud K. Gender in medicine - does it matter? Scand J Public Health. 2009;37:139-45

27. García-Chapa EG, Leal-Ugarte E, Peralta-Leal V, Durán-González J, Meza-Espinoza JP. Genetic epidemiology of type 2 diabetes in Mexican mestizos. Biomed Res Int. 2017;2017:3937893

28. Cruz M, Valladares-Salgado A, García-Mena J, Ross K, Edwards M, Ángeles-Martínez $\mathrm{J}$, et al. Candidate gene association study conditioning on individual ancestry in patients with type 2 diabetes and metabolic syndrome from Mexico City. Diabetes Met Res Rev. 2010;26:261-70.

29. Sandín M, Espelt A, Escolar-Pujolar A, Arriola L, Larrañaga I. Desigualdades de género y diabetes mellitus tipo 2: la importancia de la diferencia. Av Diabetol. 2011;27:69-105.
30. Gary-Webb TL, Suglia SF, Tehranifar P. Social epidemiology of diabetes and associated conditions. Curr Diab Rep. 2013;13:850-9.

31. Piccolo RS, Subramanian SV, Pearce N, Florez JC, McKinlay JB. Relative contributions of socioeconomic, local environmental, psychosocial, lifestyle/behavioral, biophysiological, and ancestral factors to racial/ethnic disparities in type 2 diabetes. Diabetes Care. 2016;39:1208-17.

32. Gómez-Dantés H, Fullman N, Lamadrid-Figueroa H, Cahuana-Hurtado L, Darney B, Ávila-Burgos L, et al. Dissonant health transition in the states of Mexico, 1990-2013: a systematic analysis for the Global Burden of Disease Study 2013. Lancet. 2016;388:2386-402.

33. Moreno Altamirano L, García García JJ, Soto Estrada G, Capraro S, Limón Cruz D. Epidemiología y determinantes sociales asociados a la obesidad y la diabetes tipo 2 en México. Rev Med Hosp Gen Mex. 2014;77:86-95.

34. Moreno-Altamirano L, Silberman M, Hernández-Montoya D, Capraro S, Soto-Estrada G, García-García JJ, et al. Diabetes tipo 2 y patrones de alimentación de 1961 a 2009: algunos de sus determinantes sociales en México. Gac Med Mex. 2015;151:354-68.

35. Sobers-Grannum N, Murphy MM, Nielsen A, Guell C, Samuels TA, Bishop $\mathrm{L}$, et al. Female gender is a social determinant of diabetes in the Caribbean: a systematic review and meta-analysis. PLoS One. 2015; 10:e0126799.

36. McGavock J, Wicklow B, Dart AB. Type 2 diabetes in youth is a disease of poverty. Lancet. 2017;390:1829.

37. Amin L, Shah BR, Bierman AS, Lipscombe LL, Wu CF, Feig DS, et al. Gender differences in the impact of poverty on health: disparities in risk of diabetes-related amputation. Diabet Med. 2014;31:1410-7.

38. Collier A, Ghosh S, Hair M, Waugh N. Impact of socioeconomic status and gender on glycaemic control, cardiovascular risk factors and diabetes complications in type 1 and 2 diabetes: a population based analysis from a Scottish region. Diabetes Metab. 2015;41:145-51.

39. Stringhini S, Carmeli C, Jokela M, Avendaño M, Muennig P, Guida F, et al. Socioeconomic status and the $25 \times 25$ risk factors as determinants of premature mortality: a multicohort study and meta-analysis of 1.7 million men and women. Lancet. 2017;389:1229-37. 